\title{
ON VENTCEL'S TYPE BOUNDARY CONDITION FOR LAPLACE OPERATOR IN A SECTOR
}

\author{
PETAR POPIVANOV AND ANGELA SLAVOVA
}

Communicated by Vasil V. Tsanov

\begin{abstract}
This paper deals with classical solutions of the Dirichlet-Ventcel boundary value problem (BVP) for the Laplace operator in bounded sector in the plane having opening of the corresponding angle $\varphi_{0}>0$. Ventcel BVP is given by second order differential operator on the boundary satisfying Lopatinksii condition there. As the boundary is non smooth, two different cases appear: $\frac{\pi}{\varphi_{0}}$ is irrational and $\frac{\pi}{\varphi_{0}}$ is an integer. At first we prove uniqueness result via the maximum principle and then existence of the classical solution. To do this we apply two different approaches: the machinery of the small denominators and the concept of Green function.
\end{abstract}

\section{Introduction}

This paper deals with existence and uniqueness of the classical solution for the Laplace operator equipped with Ventcel's type boundary condition in a bounded sector in the plane. Ventcel boundary conditions are second order differential conditions appearing in asymptotic models proposed by Feller and Ventcel [4], [7, 8] (interpreted as a surface diffusion). The opening of the angle $\varphi_{0}>0$ with vertex at the origin is such that $\pi / \varphi_{0} \notin \mathbb{Q}$ or $\frac{\pi}{\varphi_{0}} \in \mathbb{N}, \mathbb{Q}$ being the set of rational numbers and $\mathbb{N}$ standing for the set of positive integers. At first we state the problem and prove uniqueness result (comparison principle) via the maximum principle for elliptic equations. Our second step is to prove existence result for classical solutions. To do this we apply the machinery of small denominators. Another approach is from the theory of ordinary differential equations (see Section 3 of the paper).

Under different conditions imposed on $\varphi_{0}$ we prove existence of a $C^{2}$ solution in the bounded domain as well regularity results, including $C^{\infty}$ solutions. The solution is found in the form of convergent series in $r^{m} \sin \left(\frac{n \pi \varphi}{\varphi_{0}}\right), m, n \in \mathbb{N}$. As Laplace operator is $C^{\infty}$ and even analytic hypoelliptic, the main difficulties are in proving regularity up to the boundary. Laplace-Dirichlet-Ventcel problem in a disc, in a ring and in a bounded smooth domain were studied via pseudo-differential operators approach in [2]. 
To be more precise, we shall mention that in $1951 \mathrm{M}$. Vishik studied in a bounded smooth domain $\Omega$ the following BVP

$$
\Delta u=F \quad \text { in } \quad \Omega, \quad-\Delta^{\prime} u+\frac{\partial u}{\partial n}=f \quad \text { on } \quad \partial \Omega
$$

where $\Delta^{\prime}$ is the Laplace-Beltrami operator on $\partial \Omega$ and $n$ is the unit outer normal to $\partial \Omega$. He proved that (1) is a Fredholm BVP of index 0, i.e., it possesses finitedimensional kernel and co-kernel of the same dimension. Difficulties appear when $\partial \Omega$ has singular points (corner ponts in the plane, dihedral angles, conical points in the multidimensional case). There are a lot of investigations on the subject by V. Kondratiev, P. Grisvard, B.-W. Schulze and his collaborators and many others. We concentrate in our paper to the Dirichlet-Ventcel problem in $\mathbb{R}^{2}$ in a sector (corner domain).

\section{Formulation and Proof of the Main Results}

\subsection{Comparison Principle}

In this Subsection we shall formulate Ventcel's BVP and we shall prove a Comparison principle which guarantees the uniqueness of the solution.

The Ventcel's boundary value problem in the sector

$$
S_{R}=\left\{(r, \varphi) ; 0<r<R, \quad 0<\varphi<\varphi_{0}\right\} \in \mathbb{R}^{2}
$$

for Laplace operator is given by

$$
\Delta u=f \in C^{0}\left(\bar{S}_{R}\right),\left.\left(-\frac{\partial^{2} u}{\partial \varphi^{2}}+\alpha \frac{\partial u}{\partial n}+\beta u\right)\right|_{r=R}=0,\left.u\right|_{\varphi=0}=\left.u\right|_{\varphi=\varphi_{0}}=0 .
$$

In polar coordinates

$$
\Delta=\frac{\partial^{2}}{\partial r^{2}}+\frac{1}{r} \frac{\partial}{\partial r}+\frac{1}{r^{2}} \frac{\partial^{2}}{\partial \varphi^{2}}
$$

where the solution $u \in C^{2}\left(\bar{S}_{R}\right)$, the constants $\alpha>0, \beta>0$ and $\frac{\partial}{\partial n}=\frac{\partial}{\partial r}$ is the unit outer normal to the $\operatorname{arc} \varphi \in\left(0, \varphi_{0}\right), r=R$ of the boundary of $S_{R}$.

We propose below several useful results to be used further on.

1. Let $\alpha \geq 0, \beta \neq 0$ and $u=r^{\alpha} \sin (\beta \varphi)$. Then $\Delta u=\left(\alpha^{2}-\beta^{2}\right) r^{\alpha-2} \sin (\beta \varphi)$. Therefore, $\alpha=\beta \Rightarrow \Delta u=0, \alpha=1 \Rightarrow \Delta u=\left(1-\beta^{2}\right) \sin (\beta \varphi) r^{-1}$.

2. Let $\alpha \geq 2$. Then $\Delta\left(r^{\alpha} \log r \sin (\alpha \varphi)\right)=2 \alpha r^{\alpha-2} \sin (\alpha \varphi)$. 
3. $\sin (\beta \varphi)=0 \Longleftrightarrow \beta=\frac{k \pi}{\varphi_{0}}, k= \pm 1, \pm 2, \ldots$

To simplify the things we assume $\alpha=\beta=1$.

Proposition 1 (Comparison principle) Suppose that $\Delta u \geq 0$ in $\bar{S}_{R}, u \in C^{2}\left(\bar{S}_{R}\right)$, $\left.u\right|_{\varphi=0} \leq 0,\left.u\right|_{\varphi=\varphi_{0}} \leq 0$ and $-\frac{\partial^{2} u}{\partial \varphi^{2}}+\frac{\partial u}{\partial r}+u \leq 0$ on $r=R$. Then $u$ does not attain positive maximum in $\bar{S}_{R}$.

Proof: Put $\max _{\bar{S}_{R}} u=u\left(P_{0}\right)=M$. If $P_{0} \in S_{R}$ then $u \equiv$ const and therefore $M \leq 0$. Assume now $P_{0} \in \partial S_{R}$. If $P_{0} \in\{\varphi=0\} \cup\left\{\varphi=\varphi_{0}\right\} \Rightarrow u\left(P_{0}\right)=M$ $\leq 0$, while $P_{0} \in\left\{r=R, 0<\varphi<\varphi_{0}\right\}$ implies $-\frac{\partial^{2} u}{\partial \varphi^{2}}\left(P_{0}\right) \geq 0$ (maximum attained in an interior point of the arc, $\frac{\partial}{\partial \varphi}$ being tangential to the arc) and $\frac{\partial \varphi}{\partial n}\left(P_{0}\right)>0$ (Hopf maximum principle [5]). Thus

$$
-\frac{\partial^{2} u}{\partial \varphi^{2}}\left(P_{0}\right)+\frac{\partial \varphi}{\partial n}\left(P_{0}\right)+u\left(P_{0}\right)>0
$$

which leads to contradiction. So $u(P) \leq 0$ in $\bar{S}_{R}$.

Corollary 2. The solution of (2) is uniquely determined in $C^{2}\left(\bar{S}_{R}\right)$.

In fact, $-u$ verifies too the conditions of Proposition 1.

Proposition 3. Consider Ventcel's boundary value problem (2) with

$$
f=r^{k+1} A(r, \varphi) \quad A \in C^{2}\left(\bar{S}_{R}\right) \quad A(r, 0)=A\left(r, \varphi_{0}\right)=0, \quad k \in \mathbb{N}
$$

and assume that the solution $u$ is such that

$$
\begin{array}{cl}
u=r^{2} D(r, \varphi), \quad D \in C^{2}\left(\bar{S}_{R}\right), & D(r, 0)=D\left(r, \varphi_{0}\right)=0 \\
\frac{\pi}{\varphi_{0}}=k+2+\lambda, \quad & 0<\lambda<1 .
\end{array}
$$

Then there exists a constant $A>0$ such that for each $\varepsilon>0$

$$
|u| \leq \frac{A}{\varepsilon} r^{\frac{\pi}{\varphi_{0}}-\varepsilon} \sin \left(\frac{\pi \varphi}{\varphi_{0}}\right), \quad 0 \leq r \leq R, \quad 0 \leq \varphi \leq \varphi_{0} .
$$

Certainly, we can write $C(\varepsilon)=A / \varepsilon$.

Proof: Having in mind that $\sin \left(\frac{\pi \varphi}{\varphi_{0}}\right)>0$ for $\varphi \in\left(0, \varphi_{0}\right), A(r, 0)=A\left(r, \varphi_{0}\right)=0$ and l'Hospital rule we can write

$$
f=r^{k+1} B(r, \varphi) \sin \left(\frac{\pi \varphi}{\varphi_{0}}\right), \quad B(r, \varphi) \in C^{0}\left(\bar{S}_{R}\right)
$$


respectively $u=r^{2} E(r, \varphi) \sin \left(\frac{\pi \varphi}{\varphi_{0}}\right), E(r, \varphi) \in C^{0}\left(\bar{S}_{R}\right)$. Define now the auxiliary function

$$
u_{1}(r, \varphi)=C(\varepsilon) r^{\frac{\pi}{\varphi_{0}}-\varepsilon} \sin \left(\frac{\pi \varphi}{\varphi_{0}}\right)=C(\varepsilon) r^{k+2+\lambda-\varepsilon} \sin \left(\frac{\pi \varphi}{\varphi_{0}}\right), \quad 0<\varepsilon<\lambda<1
$$

where the constant $C(\varepsilon)>0$ will be found further on. Then

$$
\Delta u_{1}=-\varepsilon C\left(\frac{2 \pi}{\varphi_{0}}-\varepsilon\right) r^{\frac{\pi}{\varphi_{0}}-\varepsilon-2} \sin \left(\frac{\pi \varphi}{\varphi_{0}}\right)
$$

and

$$
\begin{aligned}
\Delta\left(u-u_{1}\right) & =\left[\varepsilon C\left(\frac{2 \pi}{\varphi_{0}}-\varepsilon\right) r^{k+\lambda-\varepsilon}+r^{k+1} B(r, \varphi)\right] \sin \left(\frac{\pi \varphi}{\varphi_{0}}\right) \\
& =\sin \left(\frac{\pi \varphi}{\varphi_{0}}\right) r^{k+\lambda-\varepsilon}\left[\varepsilon C\left(\frac{2 \pi}{\varphi_{0}}-\varepsilon\right)+r^{1+\varepsilon-\lambda} B(r, \varphi)\right] .
\end{aligned}
$$

Having in mind that

$$
r^{1+\varepsilon-\lambda}|B(r, \varphi)| \leq R^{1+\varepsilon-\lambda} C_{1}, \quad C_{1}=\max _{\bar{S}_{R}}|u|
$$

for $0 \leq r \leq R, 0 \leq \varphi \leq \varphi_{0}, 0<1+\varepsilon-\lambda<1$ we get that $\Delta\left(u-u_{1}\right) \geq 0$ if

$$
C(\varepsilon) \geq \frac{C_{1} R^{1+\varepsilon-\lambda}}{\varepsilon\left(\frac{2 \pi}{\varphi_{0}}-\varepsilon\right)}, \quad C_{1}=\text { const }>0 .
$$

On the other hand, $\left.u_{1}\right|_{\varphi=0, \varphi=\varphi_{0}}=\left.u\right|_{\varphi=0, \varphi=\varphi_{0}}=0$ and on $\{r=R\}$

$$
u-u_{1} \leq R^{2}\left(C_{2}-C(\varepsilon) R^{k+\lambda-\varepsilon}\right) \sin \left(\frac{\pi \varphi}{\varphi_{0}}\right), \quad C_{2}=\max _{\bar{S}_{R}}|E|>0 .
$$

Therefore,

$$
u-u_{1} \leq 0 \quad \text { on } \quad \partial S_{R} \quad \text { if } \quad C(\varepsilon) \geq \frac{C_{2}}{R^{k+\lambda-\varepsilon}} .
$$

Combining (3), (4) and the comparison principle to the Dirichlet problem for $\Delta$ operator in $S_{R}$ we get $u \leq u_{1}$ in $\bar{S}_{R}$. Similar considerations for $-u$ leads to $-u \leq u_{1} \Rightarrow|u| \leq u_{1}$ in $\bar{S}_{R}$. In other words, if $f$ vanishes of order $k+1$ with respect to $r$ and $u$ vanishes of order 2 with respect to $r$, then $u$ vanishes of order $k+2$ in $r$. Moreover, $\partial_{r}^{k+2} u \in C^{\lambda-\varepsilon}\left(\bar{S}_{R}\right), C^{\lambda-\varepsilon}, \varepsilon>0$ being the corresponding Hölder class (see also [6]). 


\subsection{Existence of Solution via Small Denominators}

This Subsection deals with the existence of a classical solution of the BVP (2) via the method of the small denominators. The angle $\varphi_{0}$ is such that $\frac{\pi}{\varphi_{0}}>2$ is an irrational number.

We assume that $0<\varphi_{0}<\frac{\pi}{2}$ as then $r^{\frac{\pi}{\varphi_{0}}} \in C^{2}[0, R]$, while $\varphi_{0}>\frac{\pi}{2} \Rightarrow r^{\frac{\pi}{\varphi_{0}}} \notin$ $C^{2}[0, R]$. We remind that each real $x=[x]+\{x\},[x]$ being the integer part of $x$, $0 \leq\{x\}<1$. We shall find a solution of (2) for right-hand side

$$
f(r, \varphi)=\sum_{m \geq 2}^{\infty} \sum_{n=1}^{\infty} B_{m n} r^{m-2} \sin \left(\frac{n \pi \varphi}{\varphi_{0}}\right) .
$$

To do this we suppose that $B_{m n}=\mathcal{O}\left(\frac{1}{\left(m^{2}+n^{2}\right)^{s}}\right)$ and apply Cauchy integral test to the double series (5) with $R \leq 1$. Then (5) is absolutely and uniformly convergent if

$$
\iint_{x^{2}+y^{2} \geq 1 / 4} \frac{\mathrm{d} x \mathrm{~d} y}{\left(x^{2}+y^{2}\right)^{s}}<\infty \Longleftrightarrow s>1 .
$$

Thus $f \in C^{0}\left(\bar{S}_{R}\right)$. Moreover, $f \in C^{1}\left(\bar{S}_{R}\right)$ for $s>\frac{3}{2}$ etc.

We split the proof of the solvability of (2) into two parts: $u=u_{1}+u_{2}$, where

$$
\begin{aligned}
\Delta u_{2}=f \quad \text { in } \quad S_{R}, & \left.u_{2}\right|_{\varphi=0}=\left.u_{2}\right|_{\varphi=\varphi_{0}}=0 \\
\Delta u_{1}=0 \quad \text { in } \quad S_{R}, & \left.u_{1}\right|_{\varphi=0}=\left.u_{1}\right|_{\varphi=\varphi_{0}}=0 .
\end{aligned}
$$

Evidently $L u_{1}=-L u_{2}$ for $r=R$, where

$$
L=-\frac{\partial^{2}}{\partial \varphi^{2}}+\frac{\partial}{\partial r}+1
$$

is the boundary operator in (2).

We look for the non unique solution of (6) in the form

$$
u_{2}(r, \varphi)=\sum_{m \geq 2} \sum_{n=1}^{\infty} A_{m n} r^{m} \sin \left(\frac{n \pi \varphi}{\varphi_{0}}\right)
$$

i.e., if

$$
\frac{\pi}{\varphi_{0}} \notin \mathbb{Q}, \quad \frac{\pi}{\varphi_{0}}>2
$$

then $m \neq n \frac{\pi}{\varphi_{0}} \notin \mathbb{Q}$, for all $m, n \in \mathbb{N}$ and therefore

$$
\Delta u_{2}=\sum_{m \geq 2} \sum_{n=1}^{\infty} A_{m n}\left(m^{2}-\frac{n^{2} \pi^{2}}{\varphi_{0}^{2}}\right) r^{m-2} \sin \left(\frac{n \pi \varphi}{\varphi_{0}}\right) \equiv f .
$$


Consequently, $A_{m n}\left(m^{2}-\frac{n^{2} \pi^{2}}{\varphi_{0}^{2}}\right)=B_{m n}$, i.e.,

$$
u_{2}(r, \varphi)=\sum_{m \geq 2} \sum_{n \geq 1} \frac{B_{m n}}{m^{2}-\frac{n^{2} \pi^{2}}{\varphi_{0}^{2}}} r^{m} \sin \left(\frac{n \pi \varphi}{\varphi_{0}}\right) .
$$

As what concerns (7) we take

$$
u_{1}(r, \varphi)=\sum_{n=1}^{\infty} A_{n} r^{\frac{n \pi}{\varphi_{0}}} \sin \left(\frac{n \pi \varphi}{\varphi_{0}}\right)
$$

beacause $\Delta u_{1}=0$. Here $A_{n}$ are unknown coefficients and one can easily see that for $r=R$

$$
\begin{aligned}
L u_{1} & =\sum_{n=1}^{\infty} A_{n}\left(\frac{n^{2} \pi^{2}}{\varphi_{0}^{2}}+\frac{n \pi}{\varphi_{0}} R^{-1}+1\right) R^{\frac{n \pi}{\varphi_{0}}} \sin \left(\frac{n \pi \varphi}{\varphi_{0}}\right) \\
& =-\sum_{m \geq 2} \sum_{n \geq 1} \frac{B_{m n}}{m^{2}-\frac{n^{2} \pi^{2}}{\varphi_{0}^{2}}}\left(\frac{n^{2} \pi^{2}}{\varphi_{0}^{2}}+m R^{-1}+1\right) R^{m} \sin \left(\frac{n \pi \varphi}{\varphi_{0}}\right)
\end{aligned}
$$

i.e.,

$$
A_{n}=-\sum_{m=2}^{\infty} \frac{B_{m n}}{m^{2}-\frac{n^{2} \pi^{2}}{\varphi_{0}^{2}}}\left(\frac{n^{2} \pi^{2}}{\varphi_{0}^{2}}+m R^{-1}+1\right) \frac{R^{m-\frac{n \pi}{\varphi_{0}}}}{\frac{n^{2} \pi^{2}}{\varphi_{0}^{2}}+\frac{n \pi}{\varphi_{0}} R^{-1}+1} .
$$

To simplify the things let $R \leq 1$. Then with $C=$ const $>0$

$$
\left|A_{n}\right| \leq C \sum_{m=2}^{\infty} \frac{1}{\left(m^{2}+n^{2}\right)^{s-1 / 2}\left|\left(m-\frac{n \pi}{\varphi_{0}}\right)\right| n^{2} R^{\frac{n \pi}{\varphi_{0}}}}
$$

and

$$
\left|u_{1}(r, \varphi)\right| \leq C \sum_{m=2}^{\infty} \sum_{n=1}^{\infty} \frac{\left(\frac{r}{R}\right)^{\frac{n \pi}{\varphi_{0}}}}{n^{2}\left(m^{2}+n^{2}\right)^{s-1 / 2}\left|\left(m-\frac{n \pi}{\varphi_{0}}\right)\right|}, \quad \frac{r}{R} \leq 1, n \geq 1 .
$$

Now we shall use the approach of the small denominators having many applications in the celestial mechanics (see for example [1]). There it is proved the following result.

Lemma 4 ([1], Chapter 3, §12) Let $\sigma>0$. Then for almost each real $\mu$ there exists a constant $K(\sigma, \mu)>0$ such that

$$
\left|\mu-\frac{p}{q}\right| \geq \frac{K}{|q|^{2+\sigma}}, \quad \text { for all } \quad p, q \neq 0, \quad p, q \in \mathbb{Z} .
$$


Evidently, $\mu \notin \mathbb{Q}$.

A result similar to (14) was proved by Liouville in 1844 for each algebraic number (non-rational). Actually A. Thue improved the theorem of Liouville in 1908, while in $1921 \mathrm{C}$. Siegel and in $1955 \mathrm{~K}$. Roth obtained the optimal in some sense results on the subject. Moreover, Liouville found effective examples of transcendental numbers, known as Liouville transcendental numbers [3]. Unfortunately, algebraic numbers form a countable set, i.e., they have zero Lebesgue measure. The real numbers $\mu$ verifying (14) have a full Lebesgue measure and almost each transcendental number is a solution of (14). Liouville numbers do not satisfy (14). We shall suppose that $\frac{\pi}{\varphi_{0}}$ is a solution of (14), i.e.,

$$
\left|\frac{m}{n}-\frac{\pi}{\varphi_{0}}\right| \geq \frac{K}{n^{2+\sigma}}, \quad \text { for all } \quad m, n \in \mathbb{N} . \Longleftrightarrow\left|m-\frac{n \pi}{\varphi_{0}}\right| \geq \frac{K}{n^{1+\sigma}} .
$$

According to (13), (15)

$$
\left|u_{1}(r, \varphi)\right| \leq \frac{C}{K} \sum_{m=2}^{\infty} \sum_{n=1}^{\infty} \frac{n^{1+\sigma}}{\left(m^{2}+n^{2}\right)^{s-1 / 2}}, \quad n^{1+\sigma} \leq C_{0}\left(n^{2}+m^{2}\right)^{\frac{1+\sigma}{2}} .
$$

The double series in the right hand side of (15) is convergent if $s>2+\frac{\sigma}{2}, \sigma>0$ and $u_{1} \in C^{0}\left(\bar{S}_{R}\right)$. The differentiability of $u_{1}, u_{2}$ is shown similarly.

This way we come to

Theorem 5. Consider the Ventcel boundary value problem (2) with right-hand side $f$ satisfying (5), $\frac{\pi}{\varphi_{0}}$ verifying (9), (15). Then for each sufficiently large $s \gg \frac{\sigma}{2}$ there exists a unique $C^{2}\left(\bar{S}_{R}\right)$ solution of (2) that can be written in the form $u=$ $u_{1}+u_{2}$ and $u_{1}, u_{2}$ satisfies (6), respectively (7).

Remark 6. The solution $u$ is given by

$$
u(r, \varphi)=\sum_{m \geq 2} \sum_{n=1}^{\infty} \frac{B_{m n}}{m^{2}-n^{2} \frac{\pi^{2}}{\varphi_{0}^{2}}} r^{m} \sin \left(\frac{n \pi \varphi}{\varphi_{0}}\right)+\sum_{n=1}^{\infty} A_{n} r^{\frac{n \pi}{\varphi_{0}}} \sin \left(\frac{n \pi \varphi}{\varphi_{0}}\right) .
$$

Evidently, $u_{1} \in C^{\infty}\left(\bar{S}_{R}\right) \Longleftrightarrow A_{n}=0, n \in \mathbb{N}$. The condition (9) implies that if $A_{1}=\ldots=A_{k-1}=0$, then $u_{1} \in C^{\left[\frac{k \pi}{\varphi_{0}}\right]}, \frac{k \pi}{\varphi_{0}}>2 k$. As $\Delta$ is hypoelliptic, $f \in C^{\infty}\left(S_{R}\right) \Rightarrow u \in C^{\infty}\left(S_{R}\right)$. In general, $f \in C^{\infty}\left(\bar{S}_{R}\right) \nRightarrow u \in C^{\infty}\left(\bar{S}_{R}\right)$.

\subsection{Existence of Solution for $\varphi_{0}=\pi$}

We shall discuss here the existence of a classical solution of (2) in the case $\frac{\pi}{\varphi_{0}} \in \mathbb{N}$ and more specifically, $\pi=\varphi_{0}$. 
As we mentioned before, another case to be investigated is $\frac{\pi}{\varphi_{0}} \in \mathbb{N}$. Let $\frac{\pi}{\varphi_{0}}=1$, i.e., $\varphi_{0}=\pi$. Again we look for $u=\tilde{u}_{1}+\tilde{u}_{2}$

$$
\tilde{u}_{1}=\sum_{n=1}^{\infty} A_{n} r^{n} \sin (n \varphi)
$$

Evidently, $\Delta \tilde{u}_{1}=0$. For appropriate $A_{n}$ the harmonic function $\tilde{u}_{1} \in C^{\infty}\left(\bar{S}_{R}\right)$. We know from the beginning of the paper that

$$
\tilde{u}_{2}=\sum_{n \neq m \geq 2} \sum_{n=1}^{\infty} A_{m n} r^{m} \sin (n \varphi)+\sum_{n=m=2}^{\infty} A_{n n} r^{n} \log r \sin (n \varphi) \equiv u_{3}+u_{4}
$$

implying

$$
\begin{aligned}
\Delta \tilde{u}_{2} & =\sum_{n \neq m \geq 2} \sum_{n=1}^{\infty} A_{m n}\left(m^{2}-n^{2}\right) r^{m-2} \sin (n \varphi)+\sum_{n=m=2}^{\infty} A_{n n} 2 n . r^{n-2} \sin (n \varphi) \\
& =f \equiv \sum_{m \geq 2} \sum_{n=1}^{\infty} B_{m n} r^{m-2} \sin (n \varphi)
\end{aligned}
$$

i.e.,

$$
A_{m n}=\frac{B_{m n}}{m^{2}-n^{2}} \quad \text { for } \quad m \neq n, \quad A_{n n}=\frac{B_{n n}}{2 n} .
$$

Consequently

$$
\left|A_{m n}\right| \leq \frac{\text { const }}{\left(m^{2}+n^{2}\right)^{s+1 / 2}}, \quad \text { for } \quad m \neq n, \quad\left|A_{n n}\right| \leq \frac{\text { const }}{n^{2 s+1}} .
$$

If $A_{m n}$ are rapidly decreasing faster than any polynomial of $\left(m^{2}+n^{2}\right)^{s}, s \in \mathbb{N}$, $s$-arbitrary, we have that $u_{3} \in C^{\infty}\left(\bar{S}_{R}\right)$. The function $r^{n} \log r \notin C^{\infty}([0, R])$ and $u_{4} \in C^{\infty}\left(\bar{S}_{R}\right)$ iff $A_{n n}=0$ for each $n \geq 2$, i.e., if $B_{n n}=0$ for $n \geq 2$. We conclude that $C^{\infty}\left(\bar{S}_{R}\right)$ solution $u$ of (2) eventually exists in the case $\pi=\varphi_{0}$ if the right-hand side $f \in C^{\infty}\left(\bar{S}_{R}\right)$ satisfies infinitely many compatibility conditions $B_{n n}=0, n \geq 2$. Due to the small denominators we have the effect of loss of regularity of the corresponding solution $u$ of (2).

\section{Some Generalizations of the Previous Results}

Consider in $T_{R_{1} R_{2}}=S_{R_{2}} \backslash \bar{S}_{R_{1}}, R_{2}>R_{1}>0$ the following BVP

$$
\begin{gathered}
\Delta u=f, \quad-\frac{\partial^{2} u}{\partial \varphi^{2}}+\frac{\partial u}{\partial n}+\left.u\right|_{S_{R_{1}} \cup S_{R_{2}}}=0 \\
\left.u\right|_{\varphi=0}=\left.u\right|_{\varphi=\varphi_{0}}=0, \quad \frac{\pi}{\varphi_{0}} \notin \mathbb{Q} .
\end{gathered}
$$


Then

$$
\left.\frac{\partial}{\partial n}\right|_{S_{R_{2}}}=\frac{\partial}{\partial r},\left.\quad \frac{\partial}{\partial n}\right|_{S_{R_{1}}}=-\frac{\partial}{\partial r} .
$$

Repeating the proof of Proposition 1 we conclude that (17) with $f=0$ possesses the unique solution $u \equiv 0$ in $C^{2}\left(\bar{T}_{R_{1} R_{2}}\right)$. As above, we are looking for $f, u$ in the form (22). Thus

$$
\begin{aligned}
u_{n}^{\prime \prime}+\frac{1}{r} u_{n}^{\prime}-\frac{n^{2} \pi^{2}}{\varphi_{0}^{2} r^{2}} u_{n} & =f_{n}(r), \quad R_{1}<r<R_{2} \\
\left.M_{1}(u)\right|_{r=R_{1}} & =-u_{n}^{\prime}\left(R_{1}\right)+\left(1+\frac{n^{2} \pi^{2}}{\varphi_{0}^{2}}\right) u_{n}\left(R_{1}\right)=0 \\
\left.M_{2}(u)\right|_{r=R_{2}} & =u_{n}^{\prime}\left(R_{2}\right)+\left(1+\frac{n^{2} \pi^{2}}{\varphi_{0}^{2}}\right) u_{n}\left(R_{2}\right)=0 .
\end{aligned}
$$

The boundary value problem (18) is simpler to deal with as

$$
r^{\frac{\pi}{\varphi_{0}}} \in C^{\left[\frac{\pi}{\varphi_{0}}\right]}([0, R]) \backslash C^{\left[\frac{\pi}{\varphi_{0}}\right]+1}([0, R])
$$

$r^{-\frac{\pi}{\varphi_{0}}}$ is unbounded near 0 , while $r^{ \pm \frac{\pi}{\varphi_{0}}} \in C^{\infty}\left(\left[R_{1}, R_{2}\right]\right)$. Certainly, the general solution of (18) is given by (we drop the subindex $n$ and put $\omega=\frac{n \pi}{\varphi_{0}}$ )

$$
u=C_{1} r^{\omega}+C_{2} r^{-\omega}+\bar{u}
$$

$C_{1}, C_{2}$ being arbitrary constants and $\bar{u}$ is some solution of the nonhomogeneous equation (18). Therefore

$$
\begin{aligned}
& C_{1} M_{1}\left(r^{\omega}\right)+\left.C_{2} M_{1}\left(r^{-\omega}\right)\right|_{r=R_{1}}=-\left.M_{1}(\bar{u})\right|_{r=R_{1}} \\
& C_{2} M_{2}\left(r^{\omega}\right)+\left.C_{2} M_{2}\left(r^{-\omega}\right)\right|_{r=R_{2}}=-\left.M_{2}(\bar{u})\right|_{r=R_{2}} .
\end{aligned}
$$

The determinant $\delta$ of the linear with respect to $C_{1}, C_{2}$ system is

$$
\begin{aligned}
\delta=\left(R_{1} R_{2}\right)^{-1}\left(\frac{R_{2}}{R_{1}}\right)^{\omega}[ & {\left[\left(\frac{R_{1}}{R_{2}}\right)^{2 \omega} \frac{\left(1+\omega^{2}\right) R_{2}-\omega}{\left(1+\omega^{2}\right) R_{2}+\omega} \cdot \frac{\left(1+\omega^{2}\right) R_{1}-\omega}{\left(1+\omega^{2}\right) R_{1}+\omega}-1\right] } \\
& \times\left(\left(1+\omega^{2}\right) R_{2}+\omega\right)\left(\left(1+\omega^{2}\right) R_{1}+\omega\right) \neq 0
\end{aligned}
$$

for $\omega>0,0<\frac{R_{1}}{R_{2}}<1$.

If we write again the subindex $n$ we easily see that

$$
\delta_{n} \sim-\omega_{n}^{4}\left(\frac{R_{2}}{R_{1}}\right)^{\omega_{n}} \quad \text { for } \quad \omega_{n}=\frac{n \pi}{\varphi_{0}} \rightarrow \infty .
$$

Again we omit the details. 


\section{Concluding Remarks}

We can generalize the existence result to the boundary value problem (2) looking for $f$, respectively $u$ in the following form

$$
f(r, \varphi)=\sum_{n=1}^{\infty} f_{n}(r) \sin \frac{n \pi}{\varphi_{0}} \varphi \quad u(r, \varphi)=\sum_{n=1}^{\infty} u_{n}(r) \sin \left(\frac{n \pi \varphi}{\varphi_{0}}\right)
$$

and supposing that $f, u$ belong to some Hölder classes. Having in mind that

$$
f_{n}(r)=\frac{2}{\varphi_{0}} \int_{0}^{\varphi_{0}} f(r, \Theta) \sin \left(\frac{n \pi \Theta}{\varphi_{0}}\right) \mathrm{d} \Theta
$$

we see that if $f \in C^{k, \alpha}\left(\bar{S}_{R}\right)$ then $f_{n} \in C^{k, \alpha}([0, R]), 0<\alpha<1$. Putting (22) in (2) we get that $u_{n}(r)$ should satisfy

$$
\begin{gathered}
u_{n}^{\prime \prime}+\frac{1}{r} u_{n}^{\prime}-\frac{n^{2} \pi^{2}}{r^{2} \varphi_{0}^{2}} u_{n}=f_{n} \quad \text { in } S_{R} \\
M u_{n} \equiv u_{n}^{\prime}(R)+\left(1+\frac{n^{2} \pi^{2}}{\varphi_{0}^{2}}\right) u_{n}(R)=0 .
\end{gathered}
$$

For the sake of simplicity denote $\omega=\frac{n \pi}{\varphi_{0}}>0$ and drop the indexes $n$ in (23). Let $0<R \leq 1$. The standard Euler substitution $r=\mathrm{e}^{t} \Longleftrightarrow-\infty<t \leq t_{0}=$ $\ln R \leq 0$ transforms the equation (23) into

$$
\frac{\mathrm{d}^{2} u}{\mathrm{~d} t^{2}}-\omega^{2} u=\mathrm{e}^{2 t} f_{1}(t), \quad f_{1}(t) \equiv f\left(\mathrm{e}^{t}\right), \quad-\infty<t \leq t_{0}
$$

having the bounded solution for $t \rightarrow-\infty$

$$
u=C_{1} \mathrm{e}^{\omega t}+\bar{u}(t), \quad C_{1}=\text { const }
$$

and $\bar{u}$ being some bounded solution of (24). Thus, $u=C_{1} r^{\omega}+\bar{u}(\ln r)$.

The change $t-t_{0}=z \leq 0 \Rightarrow z=\ln \frac{r}{R}$ transforms (24) into

$$
\frac{\mathrm{d}^{2} \bar{u}}{\mathrm{~d} z^{2}}-\omega^{2} \bar{u}=\mathrm{e}^{2 t_{0}} \mathrm{e}^{2 z} f_{1}\left(t_{0}+z\right) \equiv f_{2}(z), \quad z \geq 0 .
$$

The function $U(z)=\sinh (\omega z) / \omega$ satisfies the Cauchy problem $U^{\prime \prime}-\omega^{2} U=0$, in which $z \leq 0, U(0)=0, U^{\prime}(0)=1$. Consequently

$$
\bar{u}(z)=\int_{0}^{z} \frac{\sinh (\omega(z-\xi))}{\omega} f_{2}(\xi) \mathrm{d} \xi .
$$


The change $\xi=\ln \frac{\lambda}{R}, z=\ln \frac{r}{R}$ in the previous integral leads to

$$
\begin{aligned}
\bar{u}\left(\ln \frac{r}{R}\right) & =\frac{1}{\omega} \int_{R}^{r} \sinh \left(\omega \ln \frac{r}{\lambda}\right) f(\lambda) \lambda \mathrm{d} \lambda \\
& =\frac{1}{2 \omega}\left[r^{\omega} \int_{R}^{r} \lambda^{-\omega+1} f(\lambda) \mathrm{d} \lambda-r^{-\omega} \int_{R}^{r} \lambda^{\omega+1} f(\lambda) \mathrm{d} \lambda\right] .
\end{aligned}
$$

The kernel of (23) contains $r^{-\omega} \int_{R}^{0} \lambda^{\omega+1} f(\lambda) \mathrm{d} \lambda$ and we conclude that we can take

$$
\bar{u}=\frac{1}{2 \omega}\left[r^{\omega} \int_{R}^{r} \lambda^{-\omega+1} f(\lambda) \mathrm{d} \lambda-r^{-\omega} \int_{0}^{r} \lambda^{\omega+1} f(\lambda) \mathrm{d} \lambda\right] .
$$

Evidently

$$
r^{-\omega}\left|\int_{0}^{r} \lambda^{\omega+1} f(\lambda) \mathrm{d} \lambda\right| \leq \text { const } \cdot r^{2}
$$

while there are the possibilities $0<\omega<2, \omega \geq 2$, for the first integral in the right-hand side of (27), guaranteeing its convergence, respectively divergence for $r \rightarrow 0$. Supposing $\omega \geq 2$ (divergence to $\infty$ ) we can apply l'Hospital rule to obtain

$$
\lim _{r \rightarrow 0} r^{\omega} \int_{R}^{r} \lambda^{-\omega+1} f(\lambda) \mathrm{d} \lambda=0 .
$$

More precise results concerning the behavior of $\bar{u}$ for $r \rightarrow 0$ that take into account $f \in C^{k, \alpha}([0, R])$ and eventual vanishing of $f$ at 0 can be obtained by using Taylor formula in Hölder classes

$$
f(r)=f(0)+\frac{r}{1 !} f^{\prime}(0)+\ldots+\frac{r^{k}}{k !} f^{(k)}(0)+\mathcal{O}\left(r^{k+\alpha}\right), \quad r \rightarrow 0 .
$$

Going back to (23) we have that

$$
\left.C_{1} M\left(r^{\omega}\right)\right|_{r=R}=-\left.M(\bar{u})\right|_{r=R}
$$

i.e., the constant $C_{1}$ from (25) is uniquely determined by the equality

$$
C_{1}=\frac{-\left.M(\bar{u})\right|_{r=R}}{R^{\omega}\left(1+\omega^{2}+\omega R^{-1}\right)}
$$

We do not use in this approach series, small denominators etc., but we do not discuss the problem of the convergence of the series (22). The restriction in working in Hölder classes in $r$ are weaker than the restrictions imposed on the power series in $r$. We do not enter into technical details here. 


\section{References}

[1] Arnold V., Additional Chapters of the Theory of Ordinary Differential Operators (in Russian), Nauka, Moscow 1978.

[2] Bonnaillie-Noël, Dambrine M., Hérau F. and Vial G., On Generalized Ventcel's Type Boundary Conditions for Laplace Operator in a Bounded Domain, SIAM J. Math. Anal. 42 (2010) 931-945.

[3] Bouchschtab A., Number Theory (in Russian), Moscow 1960.

[4] Feller W., Generalized Second Order Differential Operators and Their Lateral Conditions, Illinois J. Math. 1 (1957) 459-504.

[5] Gilbarg D. and Trudinger N., Elliptic Partial Differential Operators of Second Order, Grundlehren der Math. Wissenshaften 224, Springer, Berlin 1983.

[6] Kondratiev V. and Landis E., Qualitative Theory of Second Order Linear Partial Differential Operators (in Russian), Itogi Nauki i Techniki 32, Moscow 1988.

[7] Ventcel A., Semigroups of Operators That Correspond to a Generalized Differential Operator of Second Order, Dokl. Akad. Nauk SSSR 111 (1956) 269-272.

[8] Ventcel A., On Boundary Conditions for Multidimensional Diffusion Processes, Theor. Probab. Appl. 4 (1959) 164-177.

Petar Popivanov

Institute of Mathematics and Informatics

Bulgarian Academy of Sciences

Acad. G. Bonchev Str., B1. 8

Sofia 1113, BULGARIA

E-mail address: popivano@math.bas.bg

Angela Slavova

Institute of Mathematics and Informatics

Bulgarian Academy of Sciences

Acad. G. Bonchev Str., B1. 8

Sofia 1113, BULGARIA

E-mail address: s lavova@math.bas.bg 